\title{
The Design of ADRC Based on Single Axis Stable Platform
}

\author{
Erhao Liu ${ }^{1, a}$, Hongtao Yang ${ }^{1, b}$ and Guangsen Liu ${ }^{1, c}$
}

1 Xi'an Institute of Optics and precision Mechanics CAS, Xi'an 710119, China;

aliuerhao@opt.ac.cn, byanght@opt.ac.cn, cliuguangsen@opt.ac.cn

Keywords: Permanent magnet synchronous motor (PMSM), Active disturbance rejection controller (ADRC), single axis stable platform, Simulation.

\begin{abstract}
Permanent magnet synchronous motor (PMSM) driving stable platform directly is often used in guided rockets. Due to the extended state observe (ESO) contained in the active disturbance rejection controller (ADRC), it can estimate the speed of the rotated carrier and the uncertain disturbances accurately without requiring exact mathematic model of system. An active disturbance rejection controller based on single axis stable platform is designed and optimized. The simulation results show that the system using the simplified ADRC has such characters as high robustness, high accurate and quickness.
\end{abstract}

\section{Introduction}

The stable platform can effectively isolate the motion of the carrier, produce the stable torque to offset the interference torque, and stop the relative motion to the inertial space of the stabled object, so it makes sure that the equipment installed on the platform can keep a desired attitude [1, 2]. The influence of high speed and high roll angle acceleration must be considered, when designing the single axis stable platform used in guided rockets. Good dynamic quality and steady precision is important, beyond that the single axis stable platform should have the ability of anti-interference and adaptability.

PID controller eliminates the error between target and system behavior, which don't rely on the exact mathematic model of the system. As system structures are more and more complex, and control requirements are strict, PID controller can't work effectively. In order to improve the control performance, more new control methods arise, such as robust control, adaptive control, fuzzy control, neural network control, and so on. They are limited to use because of the complex of control structures and large amount of calculations. Active disturbance rejection control technique is first proposed by Han in 1995 and its concept is "dynamic compensation linearization", which combines the core of PID controller and uses modern control theory [3, 4]. ADRC can show its advantages when realizing high precise servo control under bad environments.

The ADRC applied in the photoelectrical platform for disturbance rejection, using ESO to observe and compensate the integrated disturbance, the stable precision of visible axis could be well improved [5]. XU has been developed a single axis stable platform, which can keep stable using PID controller [6]. The stability of the sliding mode variable structure control has nothing to do with the external disturbance, LIU and YAO apply the sliding mode variable structure control into stable platform, which also improve the control performance [7, 8]. ADRC is designed for speed tracking of permanent magnet synchronous motor, which has good adaptability and robustness $[9,10,11,12]$.

This paper introduces ADRC for the single axis stable platform. This strategy consist of simplified two order position-speed ADRC and current ADRC. Simulation results show that the proposed controller can realize better dynamic response, such as faster transient and higher precision in the case of variation of the carrier disturbances than conventional PID controller.

\section{Formatting Instructions}

The mathematical model of PMSM. Assuming that the magnetic circuit is unsaturated, the hysteresis and eddy-current losses are ignored as well as that magnetic field spreads in sine, the PMSM can be expressed as follows in d-q coordinate. 


$$
\begin{aligned}
& \frac{d i_{d}}{d t}=-\frac{R_{s} i_{d}}{L_{d}}+\frac{n_{p} \omega i_{q} L_{q}}{L_{d}}+\frac{u_{d}}{L_{d}} \\
& \frac{d i_{q}}{d t}=-\frac{R_{s} i_{q}}{L_{q}}-\frac{n_{p} \omega i_{d} L_{d}}{L_{q}}-\frac{n_{p} \omega \psi_{f}}{L_{q}}+\frac{u_{q}}{L_{q}} \\
& \frac{d \omega}{d t}=\frac{K_{t} i_{q}}{J}-\frac{B \omega}{J}-\frac{T_{l}}{J}
\end{aligned}
$$

In the equation, $\omega$ is rotor angular speed, $K_{t}$ is torque constant, $i_{q}$ is q-axis stator current, $i_{d}$ is d-axis stator current, $B$ is coefficient of viscosity, $T_{l}$ is load torque, $J$ is moment of inertia, $R_{s}$ is stator resistance, $L_{q}$ is q-axis stator inductance, $L_{d}$ is d-axis stator inductance, $n_{p}$ is pole pairs, $u_{q}$ is q-axis stator voltage, $u_{d}$ is d-axis stator voltage, $\psi_{f}$ is permanent magnet flux.

The model of ADRC. ADRC includes three aspects: tracking differentiator (TD), extended state observer (ESO), and nonlinear state error feedback (NLSEF). The structure of ADRC is shown inFig.1.

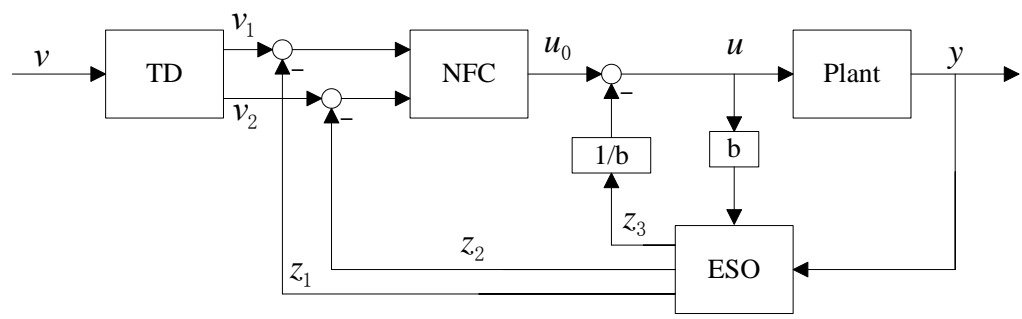

Fig. 1 The structure of ADRC

\section{A. Tracking Differentiator (TD)}

Generally a second-order tracking differentiator using symbolic function takes the following form:

$$
\left\{\begin{array}{l}
\dot{x}_{1}=x_{2} \\
\dot{x}_{2}=-r \operatorname{sign}\left[x_{1}-\vartheta(t)+\frac{x_{2}\left|x_{2}\right|}{2 a}\right]
\end{array}\right.
$$

In the equation, $x_{1}$ tracks the input signal $\vartheta(t)$ under the limit of the acceleration factor $r, x_{2}$ represents the differential of input signal $\vartheta(t)$.

\section{B. Extended State Observer (ESO)}

Consider the following system:

$$
\left\{\begin{array}{l}
\dot{x}_{1}=x_{2} \\
\dot{x}_{2}=f\left[x_{1}, x_{2}, \varpi(t), t\right]+b u \\
y=x_{1}
\end{array}\right.
$$

In the equation, $x_{1}, x_{2}$ are the state variables, $u$ is the input, $\varpi(t)$ is the external disturbance, $f\left[x_{1}, x_{2}, \varpi(t), t\right]$ denotes both the internal dynamics and the external disturbance, which is bounded. $y$ is the output.

Now let $x_{3}=f\left[x_{1}, x_{2}, \varpi(t), t\right]$, which is unknown and looked as an extended state variables. Equation (5) is linearized as follows:

$$
\left\{\begin{array}{l}
\dot{x}_{1}=x_{2} \\
\dot{x}_{2}=x_{3}+b u \\
\dot{x}_{3}=\varpi_{0}(t) \\
y=x_{1}
\end{array}\right.
$$


Here, don't care about what is $\varpi_{0}(t)$. Using $z_{1}, z_{2}, z_{3}$ estimate $x_{1}, x_{2}, x_{3}$ respectively, a nonlinear observer is designed.

$$
\left\{\begin{array}{l}
e=z_{1}-y \\
\dot{z}_{1}=z_{2}-\beta_{01} e \\
\dot{z}_{2}=z_{3}-\beta_{02} f a l\left(e, \alpha_{1}, \delta\right)+b u \\
\dot{z}_{3}=-\beta_{03} f a l\left(e, \alpha_{2}, \delta\right)
\end{array}\right.
$$

In the equation, $\beta_{01}, \beta_{02}, \beta_{03}$ are observer gains, $f a l(e, \alpha, \delta)=\left\{\begin{array}{ll}\frac{e}{\delta^{1-a}} & |e| \leq \delta \\ \operatorname{sign}(e)|e|^{a} & |e|>\delta\end{array}\right.$.

\section{Nonlinear State Error Feedback (NLSEF)}

By comparing the difference between the outputs of TD and those of ESO (shown in Fig.1), the NLSEF $u_{0}$ is used to compensate the disturbance and then drive the state trajectory to the desired reference signal. The mathematic expression for NLSEF is given as equation (8).

$$
\left\{\begin{array}{l}
e_{1}=x_{1}-z_{1}, e_{2}=x_{2}-z_{2} \\
u_{0}=k\left(e_{1}, e_{2}\right) \\
u=u_{0}-\frac{z_{3}}{b}
\end{array}\right.
$$

In the equation, $k(\square)$ represents the combination of errors.

\section{ADRC for Single Axis Stabilized Platform}

The principle of the single axis stabilized platform. The structure of the single axis stabilized

\begin{tabular}{|c|c|c|c|c|c|}
\hline \multirow{3}{*}{$\begin{array}{l}\text { Slip } \\
\text { Ring }\end{array}$} & & PMSM Stator & \multirow{3}{*}{ Encoder } & \multirow{3}{*}{$\begin{array}{c}\text { Servo } \\
\text { Controller }\end{array}$} & \multirow[b]{2}{*}{ Carrier } \\
\hline & $\begin{array}{l}\text { Platform } \\
\text { (GYRO) }\end{array}$ & PMSM Rotor & & & \\
\hline & & PMSM Stator & & & \\
\hline
\end{tabular}
platform which is driven by the PMSM is shown in Fig.2.

Fig. 2 The structure of the single axis stabilized platform

The PMSM stator is connected with the carrier, the GYRO measuring the angle and the angle velocity is fixed on the platform, which is connected with the PMSM rotor. Besides, the encoder is fixed on the other side of PMSM rotor, which is used for vector control.

The implementation of ADRC. The ADRC for the single axis stable platform is shown in Fig. 3. Where $\omega_{0}$ represents the angle velocity of the carrier, $\omega_{f b}$ is measured by GYRO, $\theta_{f b}$ is obtained by the integral of GYRO.

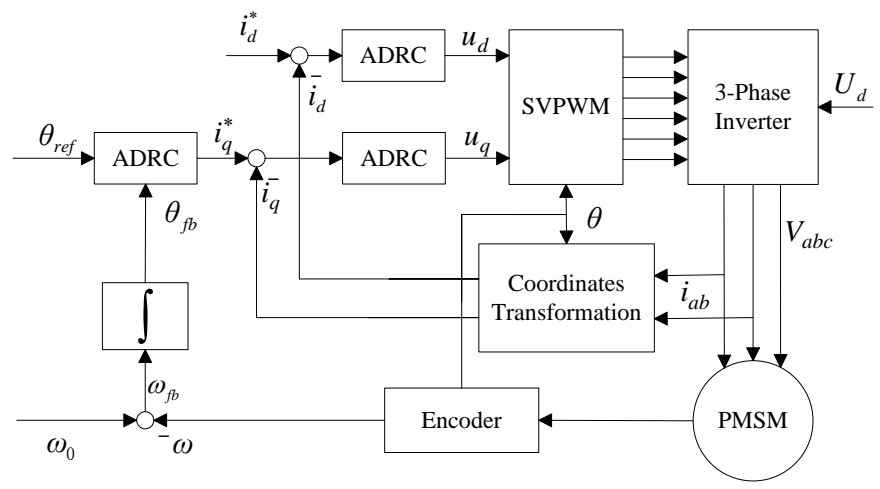

Fig. 3 The ADRC for the single axis stable platform 


\section{A. Position-Speed loop}

The TD is unnecessary for the single axis stabilized platform due to $\theta_{\text {ref }}=0$.

Now let $x_{1}=\theta_{r e f}-\theta_{f b}=-\theta_{f b}, x_{2}=\omega-\omega_{0}=-\omega_{f b}$ :

The second-order ESO for position-speed loop can be designed according to equation (5) and equation (7). $z_{1}$ tracks the angle of the platform $x_{1}, z_{2}$ tracks the angle velocity of the platform $x_{2}, z_{3}$ tracks disturbances of the system $f\left[x_{1}, x_{2}, \varpi(t), t\right], \omega_{0}$ can be observed by the ESO treated as disturbances. In the equation, $b=\frac{K_{t}}{J}, u=i_{q}^{*}, f\left[x_{1}, x_{2}, \varpi(t), t\right]=\frac{K_{t}\left(i_{q}-i_{q}^{*}\right)}{J}-\frac{B \omega}{J}-\frac{T_{l}}{J}-\omega_{0}$, include the carrier disturbances.

NLSEF is constructed as:

$\left\{\begin{array}{l}e_{1}=x_{1}-z_{1}, e_{2}=x_{2}-z_{2} \\ u_{0}=k_{1} f a l\left(e_{1}, \alpha_{1}, \delta\right)+k_{2} \text { fal }\left(e_{2}, \alpha_{2}, \delta\right)\end{array}\right.$

If the variables $\alpha_{1}=1$ and $\alpha_{2}=1$, equation (9) is degenerated of traditional PD controller, that is linear state error feedback (LSEF); otherwise, equation (9) is nonlinear PD controller, that is nonlinear state error feedback (NLSEF). The output signal of the ADRC controller is the input signal $i_{q}^{*}$ of current loop after compensating the disturbance. The simplified structure ADRC for position-speed loop is shown in Fig. 4.

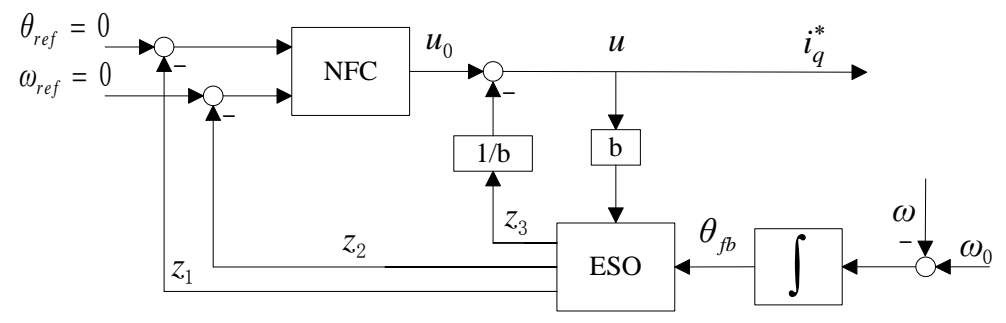

Fig. 4 Simplified ADRC for position-speed loop

In view of the design of nonlinear PD controller, the stability needs to be considered. The system becomes a two-order series system, when the compensation of ESO is executed.

$b \ddot{y}=u_{0}$

Supposing: $e_{1} P\left(e_{1}\right)=k_{1} f a l\left(e_{1}, a_{1}, \delta\right), \dot{e}_{1} Q\left(e_{1}\right)=k_{2} f a l\left(\dot{e}_{1}, a_{1}, \delta\right)$.

Equation (10) is transformed as follows:

$-b \ddot{e}_{1}=e_{1} P\left(e_{1}\right)+\dot{e}_{1} Q\left(e_{1}\right)$

Using Li Ya-pu stability theory, assuming that the Li Ya-pu function is as follow:

$V=\int_{0}^{e_{1}} e_{1} P\left(e_{1}\right) d e_{1}+\frac{1}{2} b \dot{e}_{1}^{2}$

As we all know: $e_{1} P\left(e_{1}\right)=k_{1} f a l\left(e_{1}, a_{1}, \delta\right)$ is an odd function. The positive and negative of the variable $e_{1}$ is as same as the variable $e_{1} P\left(e_{1}\right)$, so $\int_{0}^{e_{1}} e_{1} P\left(e_{1}\right) d e_{1}>0$ and $V>0$. The derivation of the equation (13):

$\dot{V}=e_{1} P\left(e_{1}\right) \dot{e}_{1}+b \ddot{e}_{1} \ddot{e}_{1}=-Q\left(e_{1}\right) \dot{e}_{1}^{2}$

$Q\left(e_{1}\right)$ is an even function and $Q\left(e_{1}\right)>0$, so $\dot{V}<0$.

Thus, the nonlinear PD controller is designed to meet the stability requirements. Where the parameters can be chosen as $k_{1}>0, k_{2}>0$.

B. Current loop

Similarly the simplified ADRC can be developed for the d-axis and q-axis current control loop. 
$\left\{\begin{array}{l}\dot{i}_{d}=f_{d}+b_{d} u_{d}, \dot{i}_{q}=f_{q}+b_{d} u_{q} \\ y_{d}=i_{d}, y_{q}=i_{q}\end{array}\right.$

In the equation, $b_{d}=\frac{1}{L_{d}}, b_{q}=\frac{1}{L_{q}}, f_{d}=-\frac{R_{s} i_{d}}{L_{d}}+\frac{n_{p} \omega i_{q} L_{q}}{L_{d}}, f_{q}=-\frac{R_{s} i_{q}}{L_{q}}-\frac{n_{p} \omega i_{d} L_{d}}{L_{q}}-\frac{n_{p} \omega \psi_{f}}{L_{q}}$.

The current loop can be treated as one-order system, so the second order ESO for $d$-axis current closed loop can be designed as:

$$
\left\{\begin{array}{l}
e_{d}=z_{d 1}-y_{d}, e_{q}=z_{q 1}-y_{q} \\
\dot{z}_{d 1}=z_{d 2}-\beta_{d 1} f a l\left(e_{d}, \alpha_{1}, \delta\right)+b_{d} u_{d}, \dot{z}_{q 1}=z_{q 2}-\beta_{q 1} f a l\left(e_{q}, \alpha_{1}, \delta\right)+b_{q} u_{q} \\
\dot{z}_{d 2}=-\beta_{d 2} f a l\left(e_{d}, \alpha_{2}, \delta\right), \dot{z}_{q 2}=-\beta_{q 2} f a l\left(e_{q}, \alpha_{2}, \delta\right)
\end{array}\right.
$$

In the equation, $z_{d 1}$ tracks $i_{d}$ and $z_{q 1}$ tracks $i_{q} ; z_{d 2}$ tracks $f_{d}$ and $z_{q 2}$ tracks $f_{q}$, which are the disturbance estimation of the motor parameters. Then the NLSEF is constructed as follows:

$$
\left\{\begin{array}{l}
u_{d}=k_{d} \operatorname{fal}\left(e_{d}, \alpha, \delta\right) \\
u_{q}=k_{q} \operatorname{fal}\left(e_{q}, \alpha, \delta\right)
\end{array}\right.
$$

If the variable $\alpha=1$, equation (16) is degenerated of traditional $\mathrm{P}$ controller, that is linear state error feedback (LSEF); otherwise, equation (16) is nonlinear P controller, that is nonlinear state error feedback (NLSEF).So the simplified ADRC for current loop is similar with the position-speed loop.

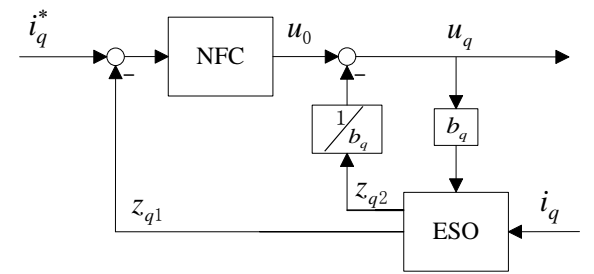

Fig. 5 Simplified ADRC for current loop

\section{Simulation}

To verify the effectiveness of the proposed ADRC method, simulation for a single axis stable platform servo system is performed and compared with the PID control. The parameters of the PMSM used in the simulation are given as Table 1.

Table 1 PMSM parameters

\begin{tabular}{ccc}
\hline Numble & Parameter & Value \\
\hline 1 & Nominal voltage $\left(U_{\mathrm{dc}}\right)$ & $24 \mathrm{~V}$ \\
2 & Nominal current $(I)$ & $4.33 \mathrm{~A}$ \\
3 & Resistance phase to phase $\left(R_{S}\right)$ & $0.307 \Omega$ \\
4 & Inductance phase to phase $\left(L_{d}=L_{q}\right)$ & $0.188 \mathrm{mH}$ \\
5 & Number of pole pairs $\left(n_{p}\right)$ & 7 \\
6 & The coefficient of viscosity $(B)$ & $0.0001 \mathrm{~N} . \mathrm{m} . \mathrm{s} / \mathrm{rad}$ \\
7 & Torque constant $\left(K_{t}\right)$ & $53.4 \mathrm{mN} / \mathrm{A}$ \\
8 & Motor inertia $(J)$ & $1210 \mathrm{~g} . \mathrm{cm}^{2}$ \\
\hline
\end{tabular}

MATLAB provides a function of constrained optimization. It's designed to solve the optimization problem under various constraints. We use this function for parameter optimization. The parameters of controller are as follows.

PID controller parameters: the position loop, $k_{p}=210, k_{i}=10$; the speed loop, $k_{p}=0.9, k_{i}=1.4$; the current loop, $k_{p}=1.5, k_{i}=310$.

ADRC controller parameters: ESO, $\beta_{01}=40000, \beta_{02}=1000000, \beta_{03}=50000000 ;$ LSEF, $k_{1}=2000, k_{2}=5$; NLSEF, $k_{1}=800, k_{2}=5$. The simulation step size: $h=0.000005$ and $h=441.32$. 
The change curve of the carrier speed is as shown in Fig.6. The load torque keeps at $0.5 \mathrm{Nm}$ in $0 \sim$ $0.35 \mathrm{~s}$. When at $0.35 \mathrm{~s}$, the load torque is raised to $0.7 \mathrm{Nm}$, the carrier speed keeps 2500rpm.

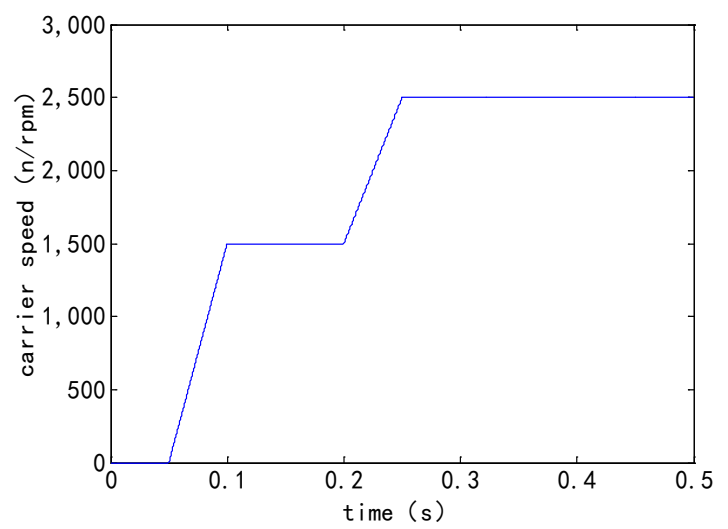

Fig. 6 The change curve of carrier speed

Comparison between ADRC controller and PID controller. Fig.7 and Fig.8 show the dynamic response and the steady response for signal axis stable platform based on ADRC controller and PID controller. ADRC controller has a more excellent performance on external disturbance rejection and dynamic response compared with PID controller.

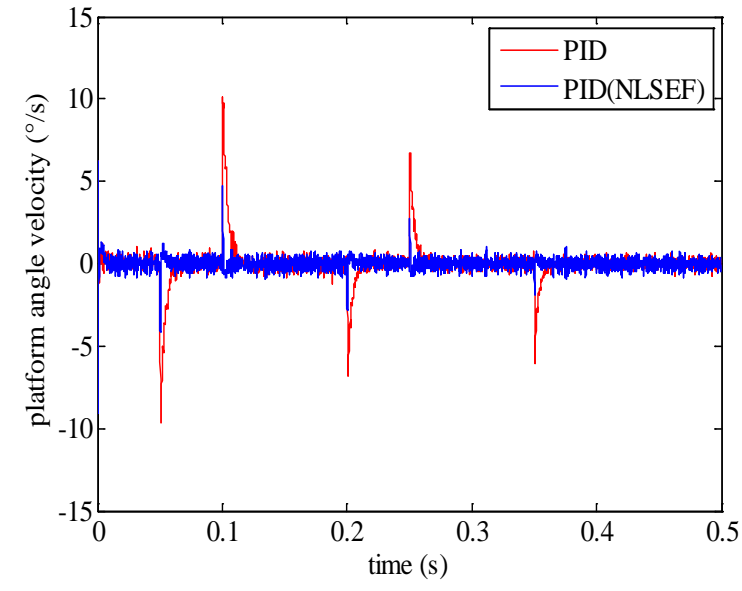

Fig. 7 Platform angular velocity

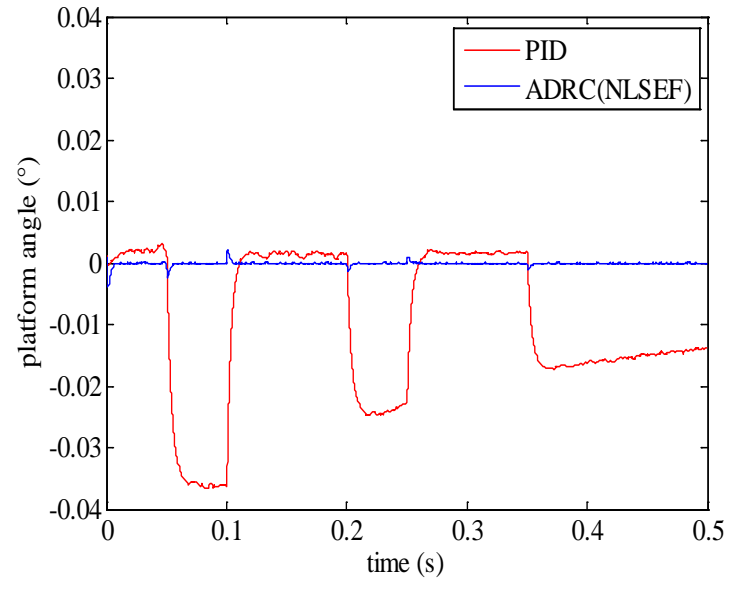

Fig. 8 Platform angle

Comparison between LSEF and NLSEF for ADRC. Fig.9 and Fig.10 show the dynamic response and the steady response for signal axis stable platform based on LSEF and NLSEF for ADRC controller. LSEF and NLSEF for ADRC have the same effect at the dynamic quality, steady precision and disturbance rejection. However, LSEF makes it easy to the setting of control parameters.

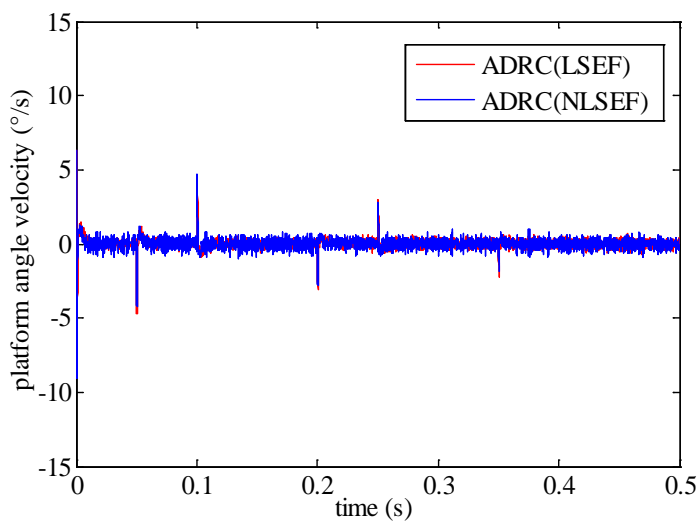

Fig. 9 Platform angular velocity

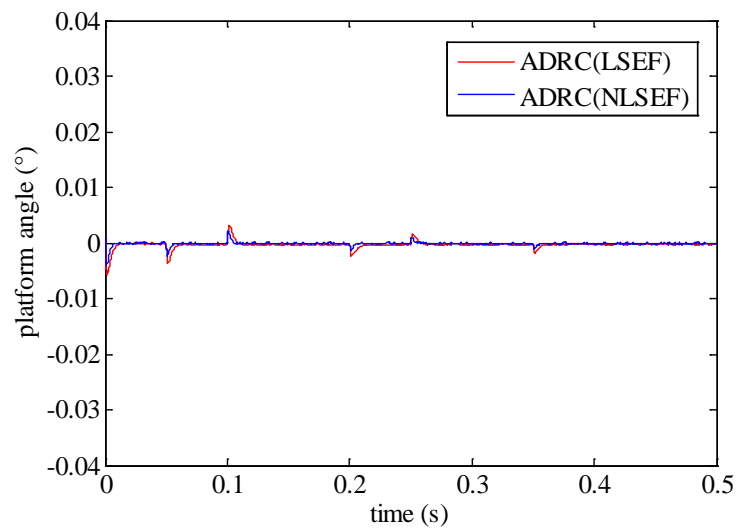

Fig. 10 Platform angle 


\section{Summary}

In this paper, the strategy of both cascade simplified ADRC has been developed for the position-speed servo control of signal axis stable platform. As verified with simulation results, it is concluded that the major advantages of the method are:

(1) ADRC strategy produces better dynamic performance and steady precision than the conventional PID controller and improves the robustness against the external carrier disturbance. What's more, a set of parameters are applicable to all various operation conditions.

(2) Simplifies the ADRC for signal axis stable platform and reduces the setting of control parameters compared with the conventional ADRC.

(3) The simplified ADRC is stable and using linear state error feedback (LSEF) replacing nonlinear state error feedback (NLSEF) can also obtain the satisfactory control performance.

\section{References}

[1] M.Masten. Inertially stabilized platforms for imaging optical systems. IEEE, Control Systems Magazine, 2008, 28(1), pp. 47-64.

[2]Hilkert J. M. Inertially stabilized platform technology Concepts and principles. IEEE, Control Systems Magazine, IEEE, 2008, 28(1), pp. 26-46.

[3] J. Q. Han. From PID to active disturbance rejection control. IEEE Trans Ind Electron, 2009, 56(3), pp. 900-906.

[4] J. Q. Han. Active Disturbance Rejection Control Technique-The Technique for Estimation and Compensation the Uncertainties. Beijing, China: Nat. Defense Ind Press, 2008.

[5]Yang Dandi, Wang Xiaobin, Hu Faxing, et al. On disturbance rejection on a photoelectrical stabilized platform. Control Conference (CCC), 2011 30th Chinese, pp. 6345-6349, 22-24 July 2011.

[6] Xu Xu Yao, Lin Hui, "Theory and design of one-axis stable platform for rotation," Electric Information and Control Engineering (ICEICE), 2011 International Conference on, pp. 4691-4693, 15-17 April 2011.

[7] LIU Er-hao, LIN Hui, LONG Jiao. The Research of Sliding Mode Variable Structure Servo Control for Single-Axis Stabilized Platform [J]. Small \& Special Electrical Motor, 2014, 04, pp. 61-63.

[8] YAO Zhao, LIU Jie, LI Yun-gong, et al. Experiment Research of Sliding Mode Variable Structure Control for GYRO Stabilized Platform. Acta Armamentarii, 2011, 32(8), pp.1019-1023.

[9]Wu Chun, Qi Rong. "The simplified active disturbance rejection control for permanent magnet synchronous motor drive system," Control Conference (CCC), 2013 32nd Chinese, pp. 4172-4176, 26-28 July 2013.

[10]Wang Lipeng, Zhang Huaguang, Liu Xiuchong. "Robust sensorless of ADRC controlled PMSM based on MRAS with stator resistance identification," Control Conference (CCC), 2011 30th Chinese, pp. 3575-3579, 22-24 July 2011.

[11]Jianping Wen, Yuchun Huang. Robust speed control of permanent magnet synchronous motor. Industrial Electronics and Applications (ICIEA), 2013 8th IEEE Conference, pp. 327-330, 19-21 June 2013.

[12]Sun Kai, Zhao Yanlei. A new robust algorithm to improve the dynamic performance on the position control of magnet synchronous motor drive. Future Computer and Communication (ICFCC), 2010 2nd International Conference, pp. V3-268, V3-272, 21-24 May 2010. 